\title{
A Design of MIMO Prototype in C-Band Frequency for Future Wireless Communications
}

\author{
Nada M. Khalil Al-Ani ${ }^{\text {* }}$, Oras A. Shareef Al-Ani ${ }^{1}$, Mahmood F. Mosleh ${ }^{1}$, \\ Read A. Abd-Alhameed ${ }^{2}$ \\ ${ }^{1}$ Computer Engineering Techniques Middle Technique University Baghdad, Iraq \\ ${ }^{2}$ School of Engineering and Informatics University of Bradford Bradford, Uk \\ *corresponding author, E-mail: eng.nada1985@ gmail . com
}

\begin{abstract}
The main challenges of MIMO design for future wireless communication is the size reduction which leads to more mutual coupling. In this paper, a proposal has presented of MIMO with four antenna elements prototype each one having a dual polarized patch rectangular microstrips. To mitigate the impact of mutual coupling, a twin of annular parasitic of a rectangular shaped and opened rib has been placed in front of each microstrip and over a circular ring slot in the ground. The advantage of such ground slot is to obtain a regular radiation pattern distributed around device body. The four elements with eight ports have integrated on $67 \times 139 \mathrm{~mm}^{2}$ PCB with FR-4 dielectric layer. The single antenna has simulated by using CST.STUDIO 2019 resulting an operated frequency of $6.23 \mathrm{GHz}$ with a band of $850 \mathrm{MHz}$ (5.81-6.66) $\mathrm{GHz}$ at $-10 \mathrm{~dB}$. The fabrication MIMO system prototype has tested to show its measurement results which are match the simulated results. All results of the MIMO prototype have operated at C-band frequency which is very important for future wireless applications.
\end{abstract}

\section{Introduction}

As it is known, the multiple-input- multiple-output (MIMO) antenna technology characterized with mitigation the multipath interference and capacity enhancement [1]. In the future pivotal wireless technology, the massive MIMO antenna (MA-MIMO) has realized fifth generation (5G) which usually ranged with minimum number started with 6 up to 100 elements or more in a single device [2]. In order to integrate a smartphone with desired quality of MIMO system, many challenges are existed [3]. Because of integrated multi elements in one space leads to more than one challenges like small size, mutual coupling and easy fabrication. To address such requirements, microstrip antenna design technique is specified with ease of fabrication, light weight and low profile [4]. Also, with exploiting the advantage of dual polarization ports of increasing the frequency spectrum can eliminate the huge size of single ports of MIMO antenna system [5]. On the other hand, the isolation can be achieved with adding a parasitic structure or modifying in the ground structure [6]. Furthermore, many researches proposed an antenna mobile operated with $5 \mathrm{G}$ applications in order to improve wave propagation [7-11]. However, some of researches deal without dual polarization which result in a huge size while others obtained narrow bandwidth, meanwhile, most of them not obtained a desired mutual coupling. In [12] propose a 5G smartphone applications with multi-ports but concerned with mutual coupling not qualified. In addition, [13] proposed MIMO antenna system with dual polarized ports which obtained a mutual coupling about $20 \mathrm{~dB}$ and a band width of $600 \mathrm{MHz}$ at $-10 \mathrm{~dB}$ and operated in sub- 6 band which qualified for $5 \mathrm{G}$ smartphone applications. In this research, as a reflected to [13] antenna structure to improve the bandwidth and mutual coupling with lesser size less size as well as it operated in C-band frequency which is a promising band for $5 \mathrm{G}$ applications. Also, to ensure the reliability of the proposed model, a fabrication part will be provided and a comparison between results of simulation and measurement will be applied.

\section{MIMO Antenna Design}

The design is started with a proposed of a single antenna element having a size of $30 \times 30 \mathrm{~mm}^{2}$ as shown in Fig. 1. It comprises of three layers; the first one is the patch layer which contained a dual polarized feeding line of $50 \Omega$ microstrip connected to power source through SMA connector. Such layer contains a twin of annular parasitic with a rectangular shape opened one of its ribs placed in front each microstrip. Placing such parasitic in this manner in order to improve the isolation between the neighboring ports. The second layer is representing the separation between patch and ground layers having a height of $1.6 \mathrm{~mm}$ and made of FR-4 with permittivity 4.4 and thermal conductivity of 0.025 . The third layer acts as a ground layer included a circular ring in a bottom of the parasitic mentioned in the patch layer. Furthermore, all dimensions detailed in Table 1 which are marked in Fig.1.

Table 1: Parameter values of the antenna design.

\begin{tabular}{cccc}
\hline parameter & value $[\mathrm{mm}]$ & parameter & value $[\mathrm{mm}]$ \\
\hline $\mathrm{W}_{\mathrm{s}}$ & 25 & $\mathrm{C}_{2}$ & 5 \\
$\mathrm{~h}_{\mathrm{s}}$ & 1.6 & $\mathrm{C}_{3}$ & 0.7 \\
$\mathrm{Lm}_{\mathrm{m}}$ & 9 & $\mathrm{r}_{1}$ & 8.7 \\
$\mathrm{~W}_{\mathrm{m}}$ & 3 & $\mathrm{r}_{2}$ & 10.2 \\
$\mathrm{C}_{1}$ & 2.5 & & \\
\hline
\end{tabular}




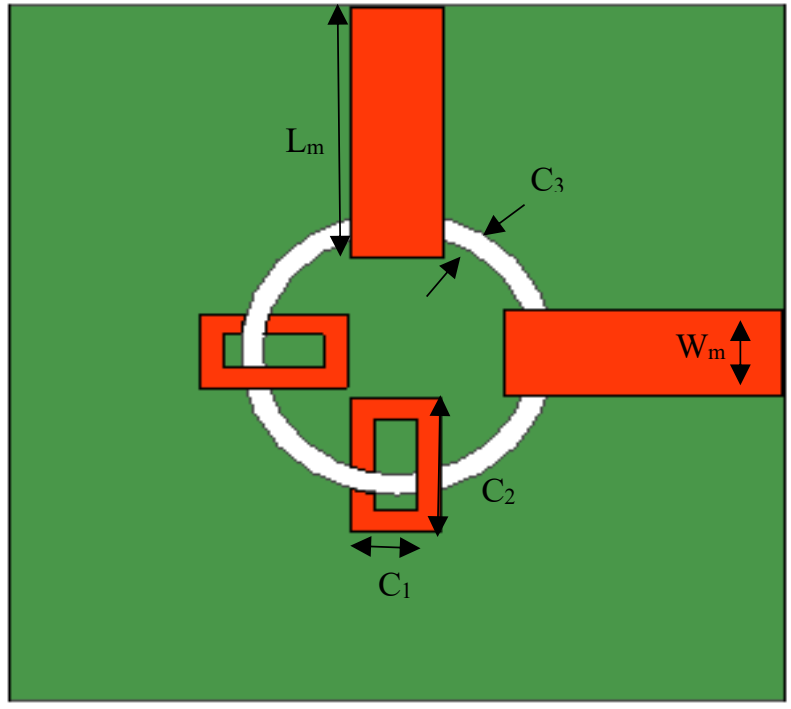

(a)

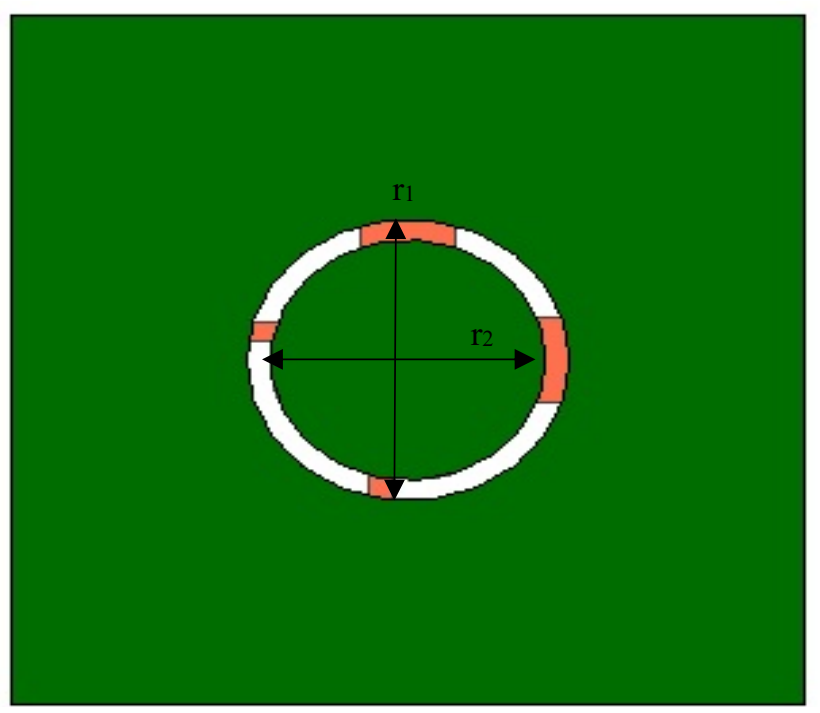

(b)

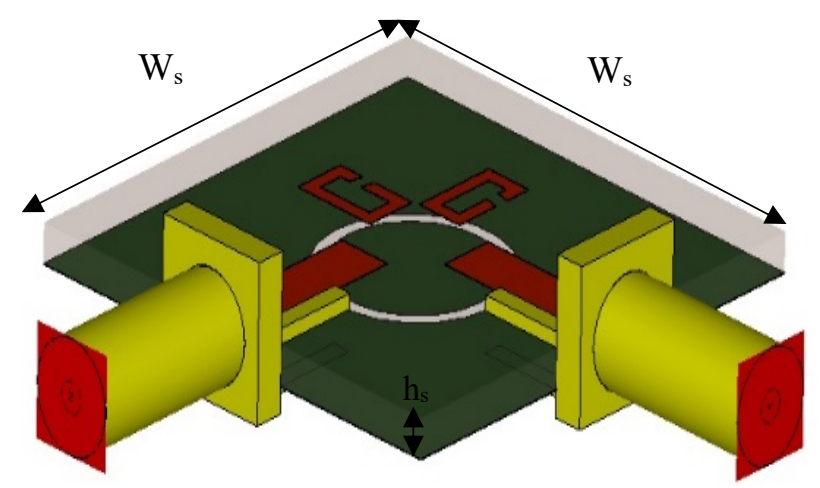

(c)

FIGURE 1: Single antenna, (a) top view, (b) bottom view, and (c) side view.
Four replicas of such proposed single antenna design have arranged on a corner of $67 \times 139 \mathrm{~mm}^{2}$ of PCB which consists of two layers of substrate and ground with same materials used in the four single antenna elements as shown in Fig.2.

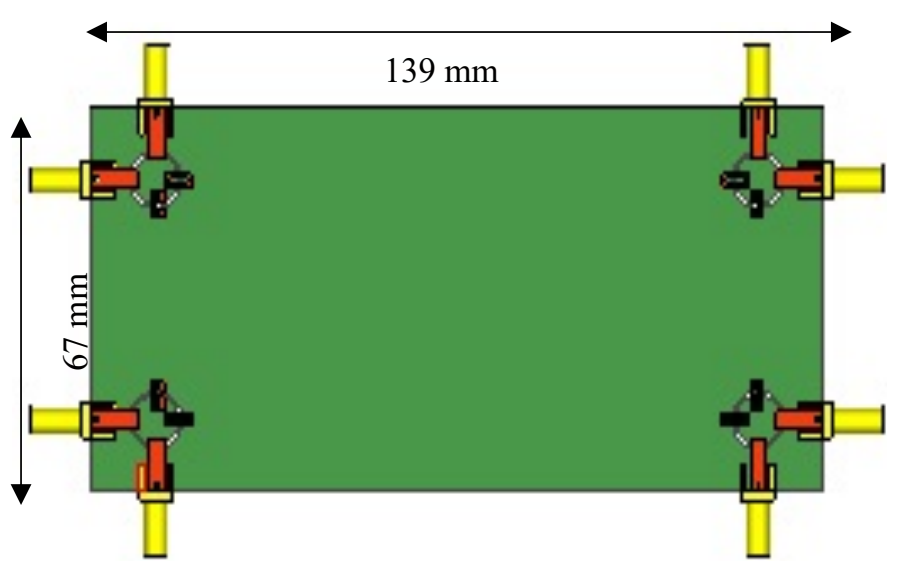

(a)

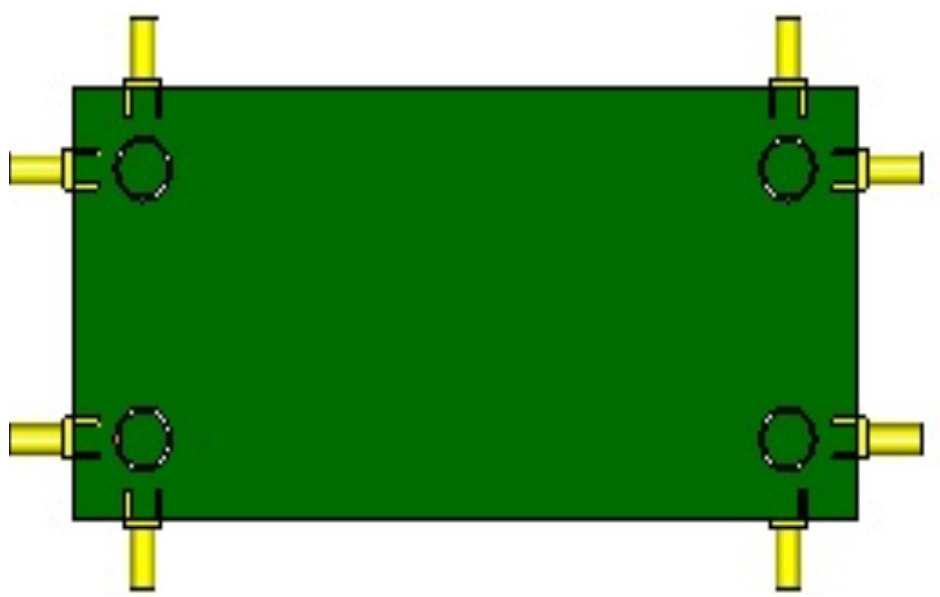

(b)

FIGURE 2: MIMIO antenna system (a) top view and (b) bottom view.

\section{Results and Discussions}

The simulation part is applied using CST.STUDIO 2019 to obtain its performance in term of return losses $S_{n m}$, mutual coupling $S_{j k}$, radiation pattern, mean effective gain (MEG), channel capacity loss (CCL), envelop correlation coefficient (ECC) and diversity gain (DG). Also, the fabrication part of MIMO antenna system has been integrated using Proto Mat S100 Laser and Electronics machine which is belong to Iraqi Ministry of Science and Technology / Electronic Manufacturing Department. In order to test the prototype of proposed MIMO antenna, has done in the laboratory of the same office mentioned above by using an Agilent N5247A vector network. The fabrication MIMO model is shown in Fig.3. The prototype of single antenna is simulated at beginning before integrated it with other antenna, the results 
are exhibit in Fig.4 which shows the operating bandwidth is $850 \mathrm{MHz}$ (5.81-6.66) $\mathrm{GHz}$ at $-10 \mathrm{~dB}$ with resonance frequency of $6.23 \mathrm{GHz}$. Such simulation shows that the return losses which represented as $\mathrm{S}_{1,1}$ and $\mathrm{S}_{2,2}$ has obtained of -20 $\mathrm{dB}$ at such operating frequency. On the other hand, the isolation between the adjacent ports which marked as $\mathrm{S}_{1,2}$ and $S_{2.1}$ has approached $-45 \mathrm{~dB}$.

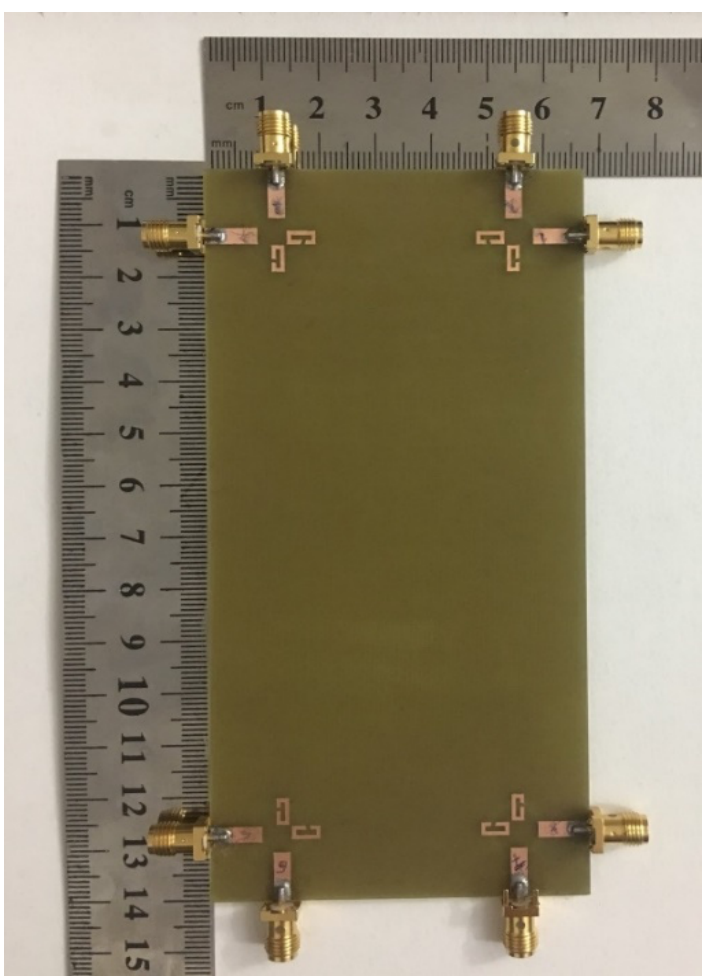

(a)

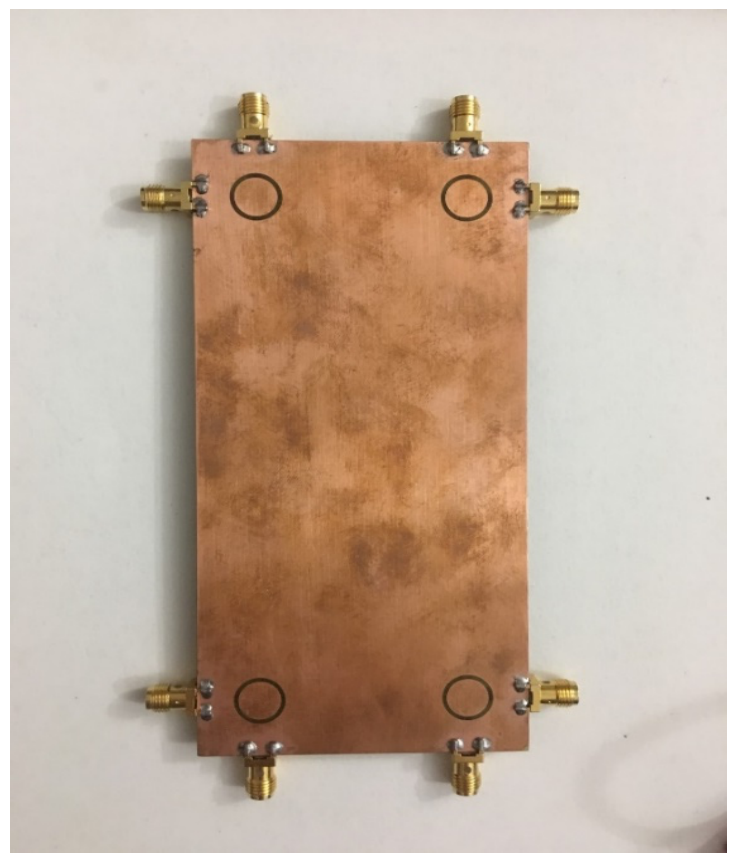

(b)

FIGURE 3: The fabrication of MIMO antenna system (a) top view and (b) bottom view

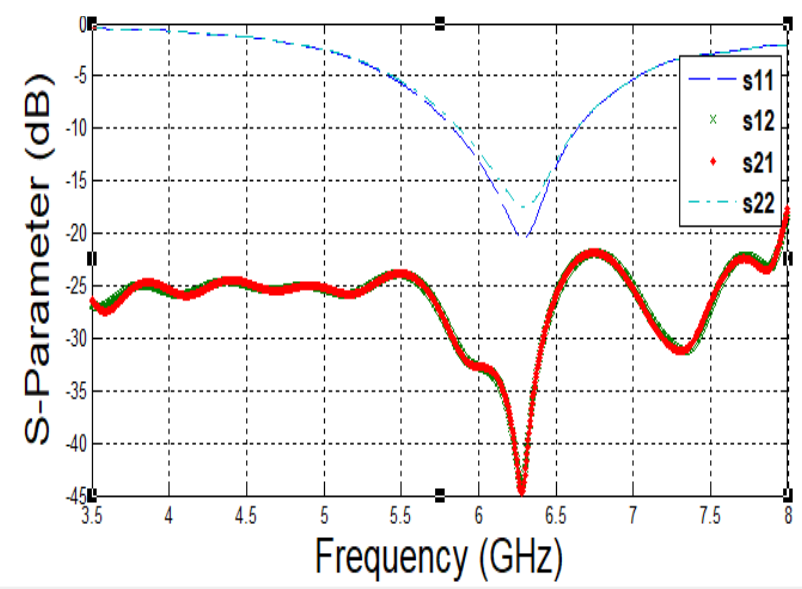

FIGURE 4: S-Parameter for single element

Fig. 5 (a) shows the results of the integrated MIMO antenna measurement which is clear in Agilent N5247A vector network analyzer screen. It is confirming the operating frequency but slightly differ by $0.23 \mathrm{MHz}$ with same band width, while the return losses less by $1 \mathrm{~dB}$ as shown in Fig. 5 (b). It is worth to mention, the capability of testing device is only test two ports and the others are locked by $50 \Omega$ FR load to avoid the mutual coupling effects. So that, all results have included only 3and 4, 5 and 6 ports. Fig.5 (c) shows the mutual coupling of testing results with compared to the simulated results. The results confirm a good matching between simulation and measurement results which indicate that the proposal can be used with currently productions.

In term of radiation pattern, Fig.6 (a) explicit how the radiation has covered all the device body in $3 \mathrm{D}$ view with $\mathrm{H}-$ max of $-22.3 \mathrm{~dB}$ and a gain of $-5.51 \mathrm{dBi}$. While Fig.6 (b) determine the radiation in $2 \mathrm{D}$ polar which obliviously seen the side lobe of $-1.1 \mathrm{~dB}$ with main lobe angle of 14.2 degree.

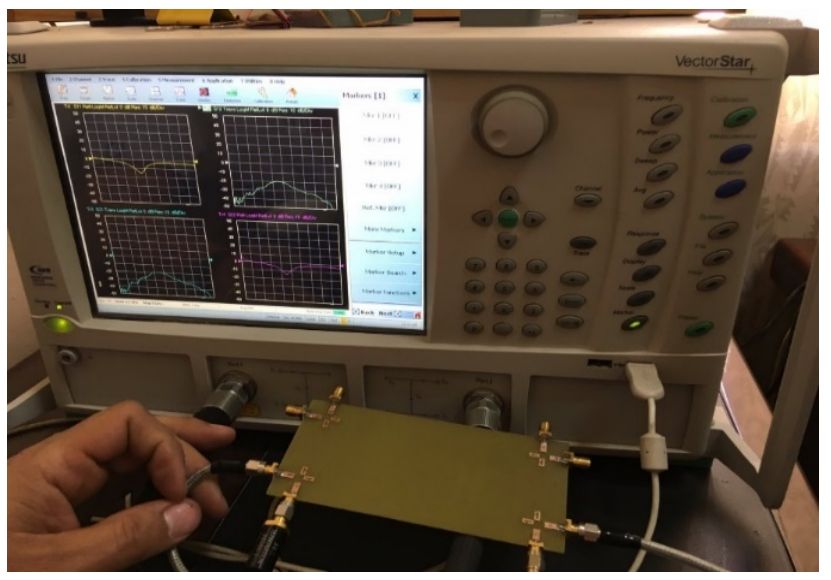

(a) 


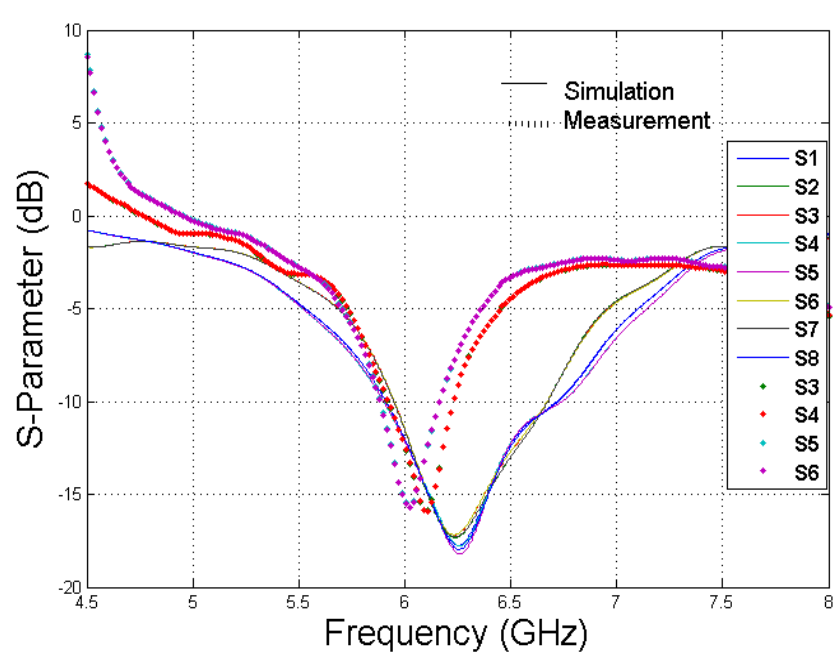

(b)

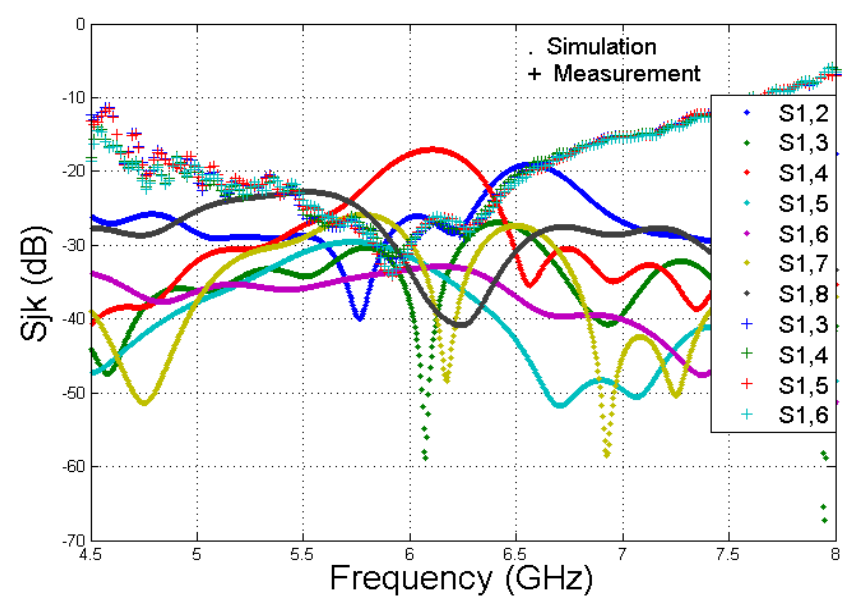

(c)

FIGURE 5: MIMO antenna system results (a) measurement screen, (b) $\mathrm{S}_{\mathrm{nm}}$ and (b) $\mathrm{S}_{\mathrm{jk}}$

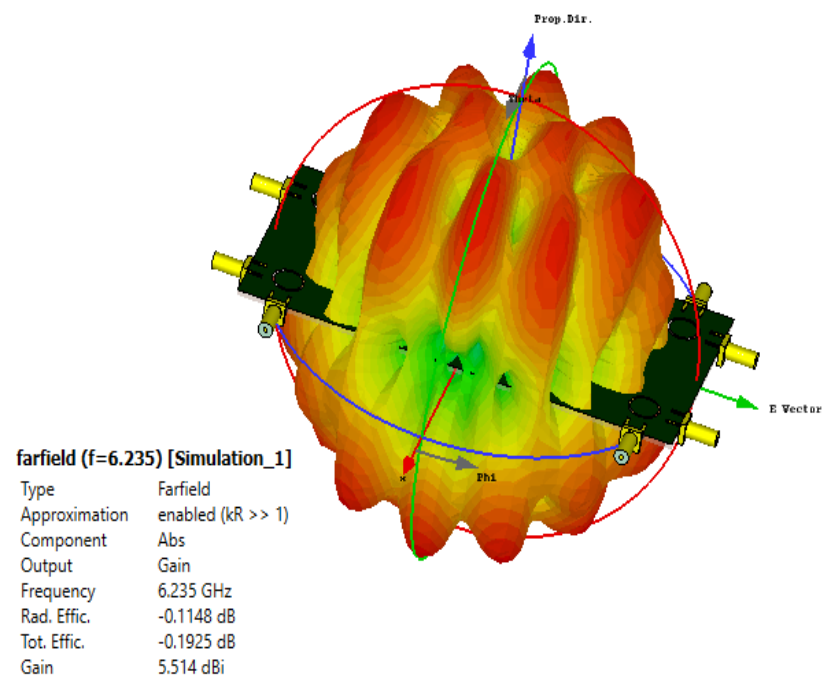

(a)

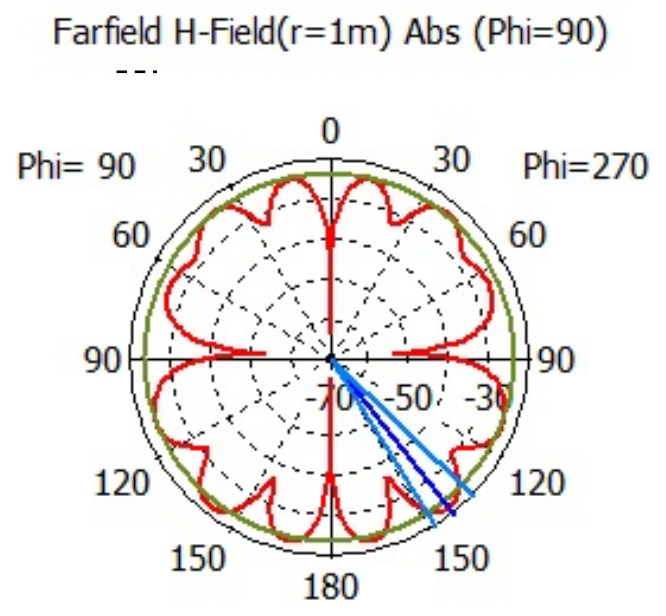

Theta / Degree vs. $\mathrm{dB}(\mathrm{A} / \mathrm{m})$

(b)

FIGURE 6: MIMO system radiation pattern (a)3D view and (b) 2D view.

\section{The performance of MIMO antenna}

In order to confirm the capability of the MIMO antenna proposal diversity, the following parameters have been calculated and analyzed:

\subsection{Envelop correlation coefficient and diversity gain}

The aim of calculating the ECC is to measure the correlation between antenna elements. In order to achieve high diversity between MIMO elements, ECC $(\rho e)$ value must be $<0.5$ which can be calculated using the radiation pattern of the far field as follow [14].

$$
\rho e=\frac{\left|\iint_{4 \pi}\left[\vec{F}_{1}(\theta, \varnothing) * \vec{F}_{2}(\theta, \varnothing) \mathrm{d} \Omega\right]\right|^{2}}{\left|\iint_{4 \pi}\left[\vec{F}_{1}(\theta, \varnothing)\right]\right|^{2} \mathrm{~d} \Omega\left|\iint_{4 \pi}\left[\vec{F}_{2}(\theta, \varnothing)\right]\right|^{2} \mathrm{~d} \Omega},
$$

where $\overrightarrow{\mathrm{F}}_{i}(\theta, \varnothing)$ is the MIMO antenna's far field property after exciting all ports. Also, Fig. 7 shows the ECC analysis over frequency which appears less than 0.001 .

On the other hand, the DG is another important parameter which must be $>9 \mathrm{~dB}$ and can be calculated related to ECC as [14].

$$
D G=10 \sqrt{1-E C C^{2}},
$$

Also. Fig. 8 displays the analysis of DG which approach $10 \mathrm{~dB}$ for all ports. 


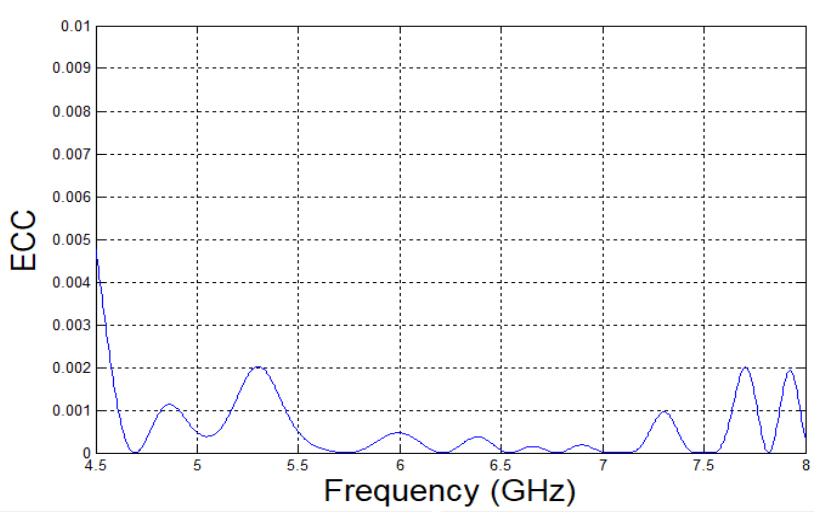

FIGURE 7: ECC of MIMO antenna elements

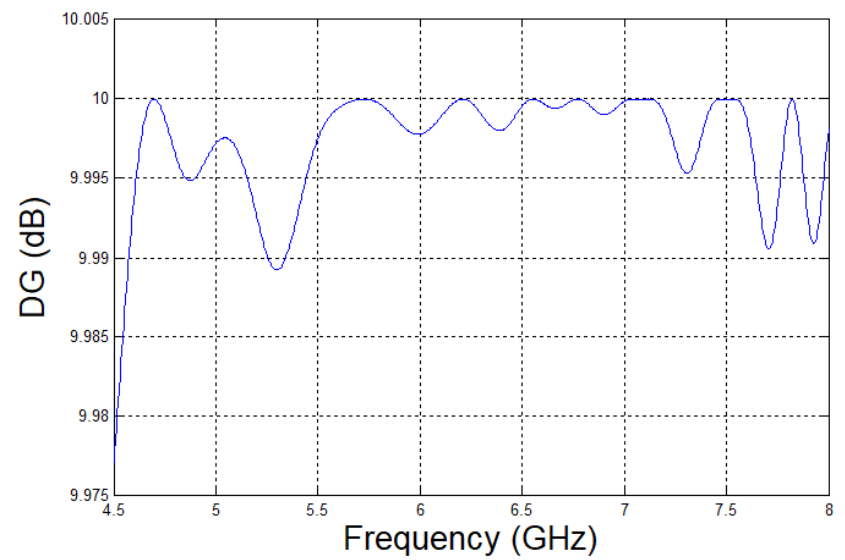

FIGURE 8: DG of MIMO antenna elements

\subsection{Channel capacity loss}

In order to evaluate the performance of MIMO antenna system, CCL must be taken in account. It is worth to mention, CCL neither increased by incrementing the bandwidth nor the transmitted power in the conventional system [15]. It just depends on the number of antenna elements with a specific assumption [15]. Also, CCL value must be $<0.4 \mathrm{bits} / \mathrm{s} / \mathrm{Hz}$ and can be calculated as [16].

$$
\begin{gathered}
C C L=-\log _{2} \operatorname{det}\left(\Psi^{r}\right), \\
\Psi^{r}=\left[\begin{array}{ll}
p 11 & p 12 \\
p 21 & p 22
\end{array}\right],
\end{gathered}
$$

where

$$
\begin{aligned}
& p_{\mathrm{ij}}=-\left(S i i^{*} S i j+S j i^{*} S i j\right) \text { for } \mathrm{i}, \mathrm{j}=1 \text { or } 2 \\
& p_{\mathrm{ii}}=1-\left(|S i i|^{2}+|S i j|^{2}\right)
\end{aligned}
$$

Fig.8 depicts the CCL analysis which observed that it achieves the conditional bound over operating frequency.

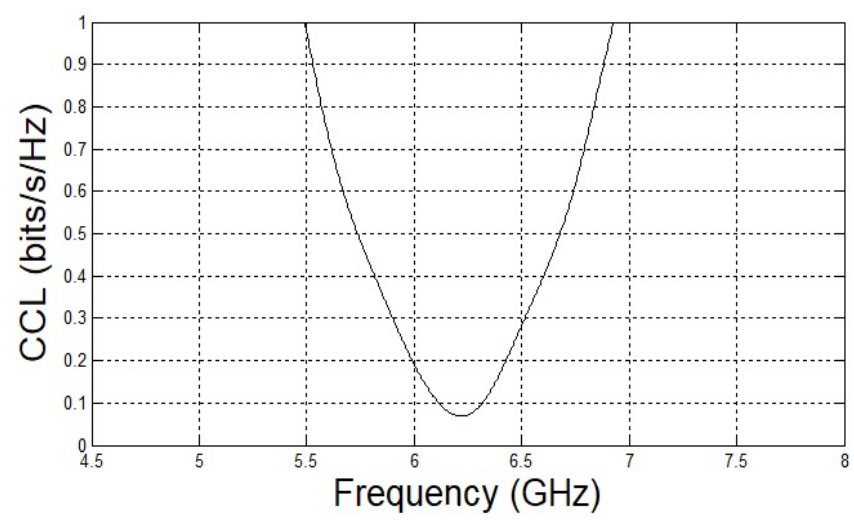

FIGURE 9: CCL of the MIMO antenna system

\subsection{Mean effective gain}

The antenna gain is measured statistically in the mobile environment by the MEG. It is defined as 'the ratio of the average power received at the antenna to the sum of the average power of the vertically and horizontally polarized waves receives by isotropic antenna "[17]. Fig. 10 shows the MEG which is less than $-3 \mathrm{~dB}$. Also, it can be calculated as [17].

$$
M E G=0.5\left[1-\sum_{j=1}^{N}|S i j|^{2}\right],
$$

where $N$ is the number of antenna element.

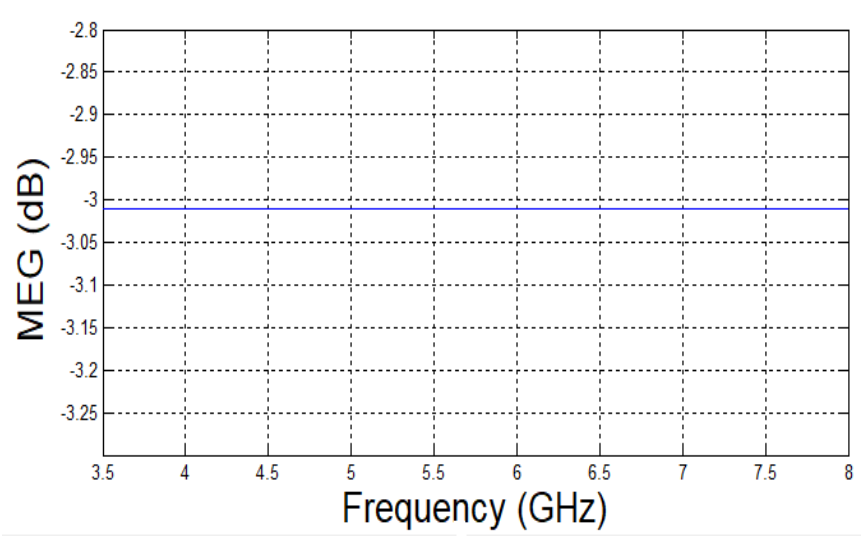

FIGURE 10: MEG of MIMO system

In order to show the proposed MIMO system performance, Table 3 shows abbreviate compression in term of bandwidth (BW), ECC, $\mathrm{S}_{\mathrm{nm}} \mathrm{S}_{\mathrm{jk}}$ between the currently proposal and other previous references. However, the proposed MIMO system operates in C-band which is the bandwidth of future communication applications. 
Table 3: Performance comparison of the MIMO

\begin{tabular}{ccccccc}
\hline ref. & $\mathrm{N}$ & $\begin{array}{c}\text { port } \\
\text { no. }\end{array}$ & $\begin{array}{c}\mathrm{BW} \\
(\mathrm{MHz}) \\
\text { at }-10 \\
\mathrm{~dB}\end{array}$ & ECC & $\mathrm{S}_{\mathrm{nm}}$ & $\mathrm{S}_{\mathrm{jk}}$ \\
\hline proposed & 4 & 8 & 850 & $<0.001$ & -18 & $<-15$ \\
{$[7]$} & 8 & 8 & 400 and & 0.01 & -16 and & -15 \\
{$[8]$} & 2 & 2 & 660 & 0.1343 & -16 & $<-15$ \\
{$[9]$} & 8 & 8 & 200 & - & -18 & -10 \\
{$[10]$} & 1 & 16 & 200 & $\cong 0.2$ & -16 & -15 \\
{$[11]$} & 4 & 8 & 200 & $<0.001$ & $\cong-15$ & $<-15$ \\
{$[13]$} & 4 & 8 & 800 & $<0.01$ & -18 & $<-15$ \\
\hline
\end{tabular}

\section{Conclusion}

In this research, a proposed MIMO prototype with four elements that each has a dual polarized port. A method of applying the parasitic rectangular is to investigate low mutual coupling. Also, a slot of circular ring has etched in the ground in reverse of the gabs made in such parasitic in order to achieve uniformly radiation pattern distribution. The proposed model has fabricated and tested to evaluate its performance. The results show that such design has operated at C-band frequency (5.81-6.66) $\mathrm{GHz}$ at -10 dB. It is worth to mention that we achieve a matching result in both simulation and measurements for in the visual and integrated of operating frequency.

\section{Acknowledgements}

Many thanks to Iraqi Ministry of Science and Technology / Electronic Manufacturing Department for their incorporating to fabricate the proposed of MIMO system and complete measurement requirements.

\section{Reference}

[1] M. Agiwal, A. Roy and N. Saxena, "Next Generation 5G Wireless Networks: A Comprehensive Survey," in IEEE Communications Surveys \& Tutorials, vol. 18, no. 3, pp. 1617-1655, thirdquarter 2016.

[2] R. Hussain, A. T. Alreshaid, S. K. Podilchak and M. S. Sharawi, "Compact 4G MIMO antenna integrated with a 5G array for current and future mobile handsets, " IET Microwaves Antennas Propag., vol. 11, no. 2, pp. 271279, 2017.

[3] H. H. Yang and Y. Q. S. Quel, "Massive MIMO meet small cell,' SpringerBriefs in Electrical and Computer Engineering. Springer, Cham: Springer, 2017. Available: https://doi.org/10.1007/978-3-319-437156 2.

[4] Mohammed Aboud Kadim, Mahmoud F. Moslah, Shereen Abdalkadum Shandal, Yaqeen Sabah Mezaal,"
New Compact Wideband Microstrip Antenna for Wireless Applications", Advanced Electromegnatic, vol.7, no. 4, pp. 85- 92, 2018.

[5] A. Dadgarpour, B. Zarghooni, B. S. Virdee, T. A. Denidni and A. A. Kishk, "Mutual Coupling Reduction in Dielectric Resonator Antennas Using Metasurface Shield for 60-GHz MIMO Systems," in IEEE Antennas and Wireless Propagation Letters, vol. 16, pp. 477-480, 2017.

[6] Queen of Mary, University of London, Antenna Designs for MIMO Systems, HMSO, 2004.

[7] Y. Li, H. Zou, M. Wang, M. Peng and G. Yang, "Eightelement MIMO antenna array for $5 \mathrm{G} / \mathrm{Sub}-6 \mathrm{GHz}$ indoor micro wireless access points," 2018 International Workshop on Antenna Technology (iWAT), Nanjing, 2018, pp. 1-4.

[8] R. Hussain, A. T. Alreshaid, S. K. Podilchak, and M.S. Sharawi, "Compact 4G MIMO antenna integrated with a 5 Garray for current and future mobile handsets," IET Microw., Antennas Propag., vol. 11, no. 2, pp. 271-279, 2017.

[9] A. A. Al-Hadi, J. Ilvonen, R. Valkonen, and V. Viikari, "Eight-element antenna array for diversity and MIMO mobile terminal in LTE $3500 \mathrm{MHz}$ band,' Microw. Opt. Technol. Lett., vol. 56, no. 6, pp. 1323-1327, 2014.

[10] K. Wong, J. Lu, L. Chen, W. Li, Y. Ban and C. Li, "16Antenna array in the smartphone for the 3.5-GHz MIMO operation," 2015 Asia-Pacific Microwave Conference (APMC), Nanjing, 2015, pp. 1-3.

[11] L. Sun, H. Feng, Y. Li and Z. Zhang, "Compact 5G MIMO Mobile Phone Antennas with Tightly Arranged Orthogonal-Mode Pairs," in IEEE Transactions on Antennas and Propagation, vol. 66, no. 11, pp. 63646369, 2018.

[12] Y. Li, C. Sim, Y. Luo and G. Yang, "Multiband 10Antenna Array for Sub-6 GHz MIMO Applications in 5G Smartphones," in IEEE Access, vol. 6, pp. 2804128053, 2018.

[13] N. O. Parchin et al., "Eight-Element Dual-Polarized MIMO Slot Antenna System for 5G Smartphone Applications," in IEEE Access, vol. 7, pp. 15612-15622, 2019.

[14] Adamu Halilu Jabire, Hong-Xing Zheng, Anas Abdu and Zhiwei Song, "Characteristic Mode Analysis and Design of Wide Band MIMO Antenna Consisting of Metamaterial Unit Cell," Electronics, vol. 8, no. 68, 2019.

[15] Chen-Nee Chuah, D. N. C. Tse, J. M. Kahn and R. A. Valenzuela, "Capacity scaling in MIMO wireless systems under correlated fading," in IEEE Transactions on Information Theory, vol. 48, no. 3, pp. 637-650, March 2002.

[16] Wael A.E. Ali and Ahmed A. Ibrahim, “A compact double-sided MIMO antenna with an improved isolation for UWB applications," Int. J. Electron. Commun. (AEÜ), vol.82, pp. 7-13, 2017.

[17] Nasir J., Jamaluddin M.H., Ahmad Khan A., Kamarudin M.R., Leow C.Y. and Owais O., "Throughput Measurement of a Dual-Band MIMO Rectangular Dielectric Resonator Antenna for LTE Applications," 
Sensors, vol. 17, no.1, pp. 148-163, 2017. Available: https://doi.org/10.3390/s17010148. 